\title{
The effect of residual stresses on the strain evolution during welding of thin-walled tubes
}

\author{
Maarten De Strycker ${ }^{1, a}$, Wim Van Paepegem ${ }^{2, b}$, Luc Schueremans ${ }^{3, c}$ and \\ Dimitri Debruyne $e^{4, d}$ \\ ${ }^{1}$ MeM2P research group, Catholic University College Ghent, Gebroeders Desmetstraat 1, Ghent, \\ Belgium \\ ${ }^{2}$ Department of Materials Science and Engineering, Ghent University, St.-Pietersnieuwstraat 41, \\ Ghent, Belgium \\ ${ }^{3}$ Department of Civil Engineering, KULeuven, Kasteelpark Arenberg 40, Heverlee, Belgium \\ ${ }^{4}$ Department MTM, KULeuven, Kasteelpark Arenberg 44, Heverlee, Belgium \\ ${ }^{a}$ Maarten.DeStrycker@kahosl.be, ${ }^{b}$ Wim.VanPaepegem@ugent.be, \\ 'Luc.Schueremans@bwk.kuleuven.be, 'Dimitri.Debruyne@mtm.kuleuven.be
}

Keywords: Residual stresses, stainless steel, tube, welding

\begin{abstract}
In many applications, negative effects of residual stresses in the material stemming from the production process, are regularly encountered. These residual stresses in cold-rolled steel tubes are mainly due to two mechanisms: (i) the rolling of the flat plate into a circular cross-section and (ii) afterwards closing this section with a weld bead. This research focuses on the residual stresses due to the welding process. In an experimental setup abstraction is made of the real production process of the tube. A finite element model is built of this experimental setup. Validation of the welding simulations is done by comparing the strain evolution in both the experiment and the simulation. In this validation process, sometimes a discrepancy between the measured strain evolution and the one obtained from the numerical analysis is seen. In this contribution it is numerically investigated how initial residual stresses affect the thermal strain evolution in the tube during the welding process. This is done in two ways: firstly an initial stress field in hoop direction, based on the spring back of the tube when cut is taken as the reference state and secondly the stress/strain state after the first weld is used in stead of the virgin material state. The conclusion for both assumptions is that the strain evolution during the welding is affected by the initial stress/strain state of the material.
\end{abstract}

\section{Introduction}

When a tube is machined using laser cutting, but also in other applications of tubes, negative effects of residual stresses in the material are regularly encountered. These residual stresses in cold-rolled steel tubes are mainly due to two mechanisms: (i) the rolling of the flat plate into a circular crosssection and (ii) afterwards closing this section with a weld bead. The first production step is causing plastic deformation, the second can be viewed as a non-uniform heating and cooling cycle on the material, preventing the elastic spring back of the bent material.

In order to estimate the residual stress after the welding of a tube, a finite element analysis (FEA) of the welding process is performed. FEA has the advantage that the residual stress/strain state in the tube as a whole can be determined and can be used later on in cutting simulations. This analysis is very dependent on numerous input parameters such as thermo-mechanical temperature-dependent material properties, thermal load definition, thermal and mechanical boundary conditions. All of these parameters are introduced in the model with a certain error and it is thus important to check the FEA results. 
Validation of the FEA results can be done in several ways e.g. the residual stresses could be measured directly, but in this research the strain development during the welding is used as a validation tool. Whereas the first method allows only a validation of the final state in some discrete points, the second method allows a continuous observation during the welding process and over a certain area of the tube as strains are measured with the digital image correlation (DIC) technique.

A comparison of the experimental data with numerical results sometimes reveals a discrepancy between the measured strain evolution and the strain curve obtained from the numerical analysis. Apart from mentioned error sources (poorly known material parameters and the use of approximate boundary conditions), the initial stress/strain state of the tube, can be considered as a culprit for the observed strain discrepancies. The welding simulations are started from a tube in a virgin stress/strain state as the residual stresses due to the welding and rolling are actually not known. In the experimental setup however, a finished tube is welded once more. The material in the experimental setup contains as such already residual stresses due to rolling and welding.

In this contribution it is numerically investigated how initial residual stresses affect the thermal strain evolution in the tube during the welding process. This is done in two ways: (i) an initial stress field in hoop direction is implemented corresponding to the spring-back when the tube is cut in longitudinal direction and (ii) an initially stress-free tube is subjected twice to the thermal cycle corresponding to the welding of the tube.

\section{Description of the tube and the FEA model}

The tube under consideration is an austenitic stainless steel (EN 1.4301) tube with a diameter of $60 \mathrm{~mm}$ and a wall thickness of $1.5 \mathrm{~mm}$. The length of the tube is $400 \mathrm{~mm}$ and a weld bead with length of $300 \mathrm{~mm}$ is put on top in longitudinal direction. These lengths are chosen with the intention of representing the real, continuous production process. This means with a continuous stress/strain state over a substantial length of the tube without effects of the starting and stopping of the weld bead. The length of the weld bead is shorter than the tube due to practical considerations in the experimental setup. In the experimental setup, the gas tungsten arc welding (GTAW) torch is held by a robot, assuring controlled welding conditions: constant heat-input, welding speed and distance between torch and work piece. A sketch of the setup is given in Fig. 1.

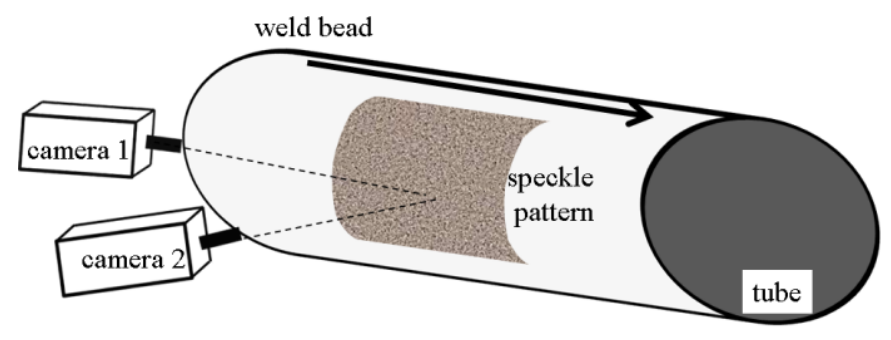

(a)

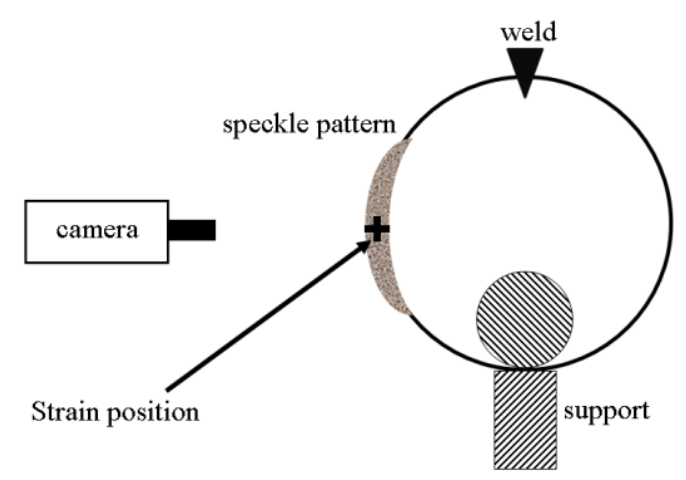

(b)

Fig. 1: sketch of the setup of the tube with the DIC cameras and indication of the circumferential position where the strain is followed

In both the experimental setup and the simulation, abstraction is made of the actual welding process of the tube. While on the production line, the tube is welded by passing it through a high frequency induction welding coil or under a GTAW torch, in this research the tube is kept static and the heat source is moving. The tube used here is a completely finished tube as delivered by the manufacturer. This means that the material has been rolled and welded before the experiment is started. Without going into details about the experimental setup, the strains are measured with the digital image correlation (DIC) technique [1-4]. That is why the cameras and the speckle pattern are 
shown in Fig. 1. It has been shown before that DIC is an appropriate technique for following strain evolution in this welding setup [5]. The experimental and the numerical results are compared in similar positions and over equal areas, following from the strain gauge position and dimensions: the hoop strain is followed in a zone of $3 \times 6 \mathrm{~mm}^{2}$ (orientated in hoop direction), 190mm from the weld start; the longitudinal strain is followed in a zone of $6 \times 3 \mathrm{~mm}^{2}$ (orientated in longitudinal direction), $225 \mathrm{~mm}$ from the weld start.
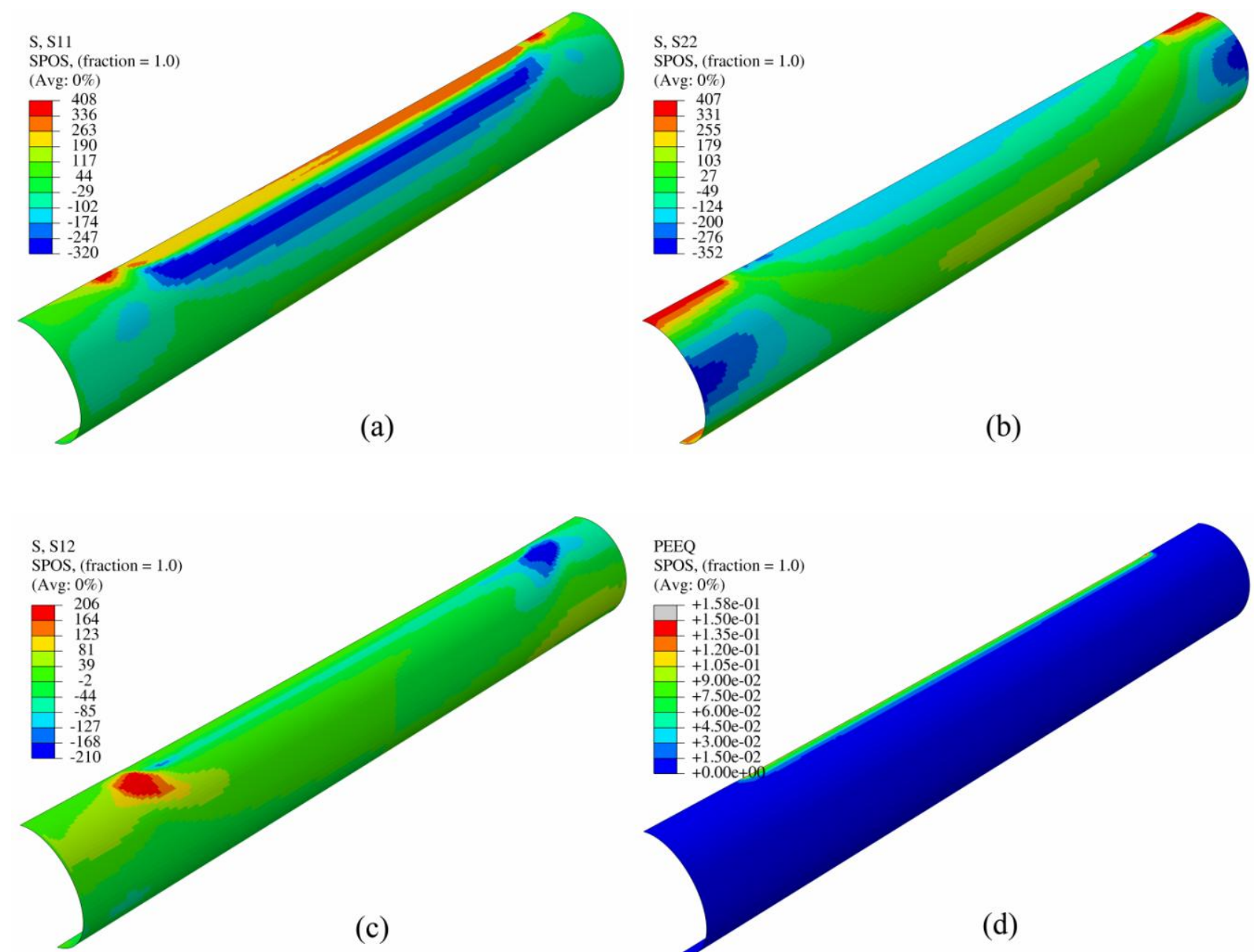

Fig. 2: stress/strain state after the first welding cycle: (a) normal stress in the longitudinal direction; (b) normal stress in circumferential direction; (c) in-plane shear stress; (d) Plastic Equivalent Strain. All variables are plotted in the section point on the outer surface of the tube, stresses are given in MPa.

The geometry, boundary conditions and loads are symmetric, thus only half of the tube is modelled in the Abaqus/standard [6] model. As is common in modelling welding processes, the mechanical analysis, in which stresses and strains are calculated, is done separately from the thermal analysis, which calculates the temperature distribution in the welded work piece. The geometry is built with linear, quadrilateral shell elements: in the thermal analysis elements with five section points through the thickness (DS4 elements [6]) are chosen based on earlier simulation studies [7] and in the mechanical analysis elements with reduced integration and five section points (S4R elements with one integration point [6]) have proven to give reliable results. In circumferential direction, the mesh is made finer at the weld bead and coarser towards the support of the tube, in longitudinal direction, the elements are evenly spaced. The model contains about 20000 elements in total. Temperature-dependent material properties (elasto-plastic behaviour, density, thermal conductivity, thermal expansion, specific heat capacity, latent heat between the solidus and the liquidus phase) are based on available literature and experimentally measured values [4,8-10]. The 
austenitic steel does not show any phase transformations between room temperature and the melting point [11]. In the current model, no specific material model for the molten material is adopted. As the temperature rises, the material looses strength and stiffness. Above the melting temperature $\left(1400^{\circ} \mathrm{C}\right)$, the yield stress is about $10 \%$ of the yield stress at room temperature.

In the thermal analysis, the heat input of the welding torch is modelled with the double-ellipsoid as described by Goldak [12] and implemented in the model with the DLFUX user subroutine [6]. The double-ellipsoid describes the distribution of the heat generated by the torch, the total energy generated in the welding process can be calculated as the current multiplied by the voltage over the weld beam. These two variables can be read out from the welding machine. The efficiency of the welding process, i.e. how much of the generated heat is absorbed by the material, is on this moment uncertain. An efficiency of $85 \%$ is assumed in the current model. The heat flows out of the model by radiation and convection to the ambient space and conduction to the support. It is obvious that this temperature dependent input is a cause of uncertainty in the model. An optimization method on the uncertain parameters in the thermal analysis (convection, emissivity and efficiency of the welding process) combined with reliable temperature measurements can bring the temperature field in better agreement with the actual temperatures in the tube during the welding. This consideration does not fall within the scope of this contribution and is not relevant for the current conclusions. The thermal analysis consists of 3 steps: first, to form a melting pool, the torch is kept still for $3 \mathrm{~s}$, then the weld bead is laid on the tube, which takes 150 s and finally the tube is cooled down to the ambient temperature. The total time to finish the simulation is $1200 \mathrm{~s}$.

Based on the temperatures calculated in the thermal analysis, stresses and strains are calculated in the mechanical analysis. This analysis step allows following the stress/strain evolution in the model during the welding and reveals the residual stresses in the tube at the end of the welding process.

The residual stresses after the welding are shown in Fig. 2. The high residual tensile stresses in longitudinal direction (Fig. 2 (a)) indicate clearly the location of the weld bead. Plastic deformations seem to occur only in the neighbourhood of the weld bead (Fig. 2 (d)).

\section{Stress source I: elastic spring back in hoop direction}

To have an idea about the residual stress in hoop direction, a slice of the tube is cut parallel to the longitudinal axis. The residual stresses in hoop direction make the slice spring open. For the tube under consideration, the tube springs open by about $2.5 \mathrm{~mm}$. This value is corrected for the width of the cutting blade. This opening is attributed to elastic bending stresses in hoop direction.

The shell element used in this model has 5 section points through the thickness. These residual stresses are imposed by defining an initial stress field that represents pure bending conditions in hoop direction. It was implemented in the model as follows: the inner section point is assigned a stress of $-70 \mathrm{MPa}$, the second section point $-35 \mathrm{MPa}$, the middle section point is stress-free, the fourth is assigned $+35 \mathrm{MPa}$, and the outer section point has a stress of $+70 \mathrm{MPa}$. This stress field is imposed on all the elements at the beginning of the analysis. It was checked with a simulation that this initial stress field creates a bending moment that opens the tube $2.5 \mathrm{~mm}$ when the symmetry boundary condition is removed from the model.

The results of the simulation are presented in Fig. 3. The strain evolution plotted here is for the strain point indicated in Fig. 1, which is at $90^{\circ}$ in the cross section, seen from the weld bead. From these graphs it is clear that an initial stress field has an effect on the strain evolution in hoop direction (Fig. 3(a)). It is concluded that implementing this stress field brings the strain evolution as calculated with FEA closer to the strain evolution as measured with DIC. As expected, the evolution of the strain in the longitudinal direction is however not affected by this initial stress field.

The method used to obtain the stress in circumferential direction is rather rudimentary, but the simulation results show that the small stresses have a substantial influence on the result. Estimating the stress field in longitudinal direction cannot be done in such a simple way. For both stress 
components applies that they can be obtained by simulating the entire roll forming process of the tube from flat plate into circular cross section.

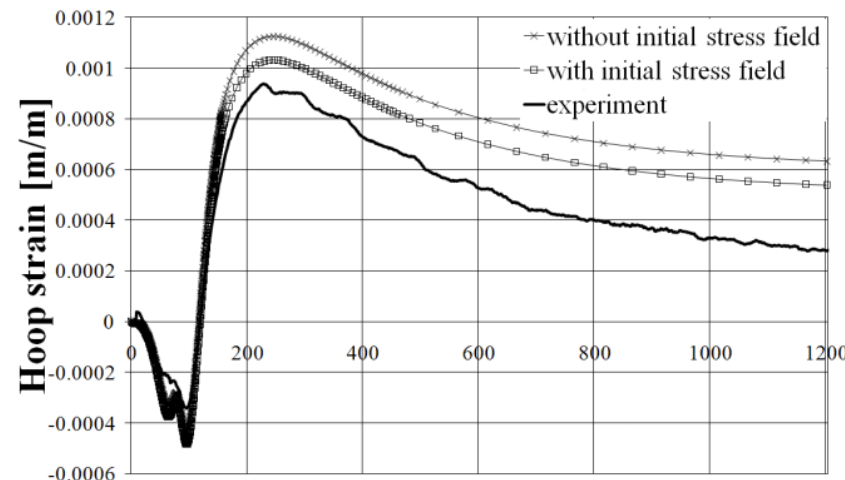

Time [s]

(a)

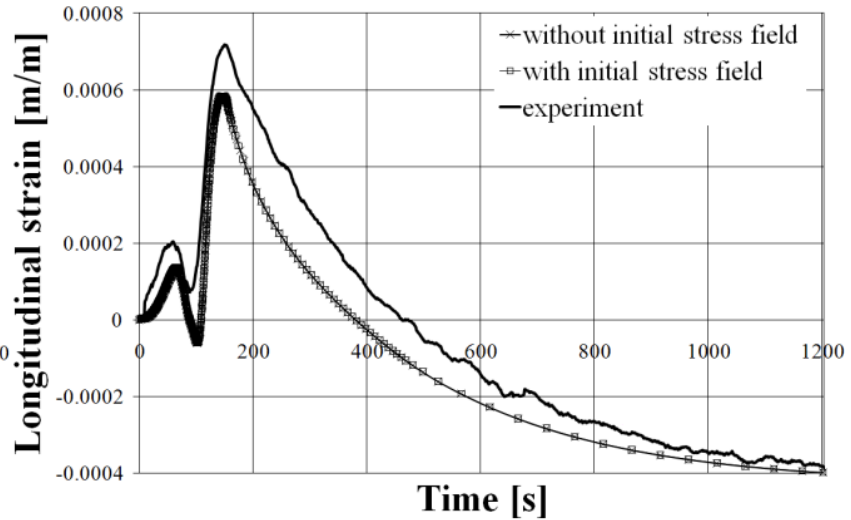

(b)

Fig. 3: Strain evolution obtained in the experiment and by simulation without and with initial stress field: (a) in hoop direction; (b) in longitudinal direction.

\section{Stress source II: the tube subjected to a second thermal load cycle}

The tubes used in the experimental setup are in the state as produced by the manufacturer. This means that the welding in the experimental setup is in fact a second welding imposed on the tube. In this section it is investigated whether imposing two times the same heating and cooling cycle has an effect on the strain evolution observed in the experiment. The temperature evolution as calculated in the thermal analysis is applied two times consequently in the same mechanical analysis, and in that way the residual stress/strain state after the first thermal cycle is the starting state in the second thermal cycle.

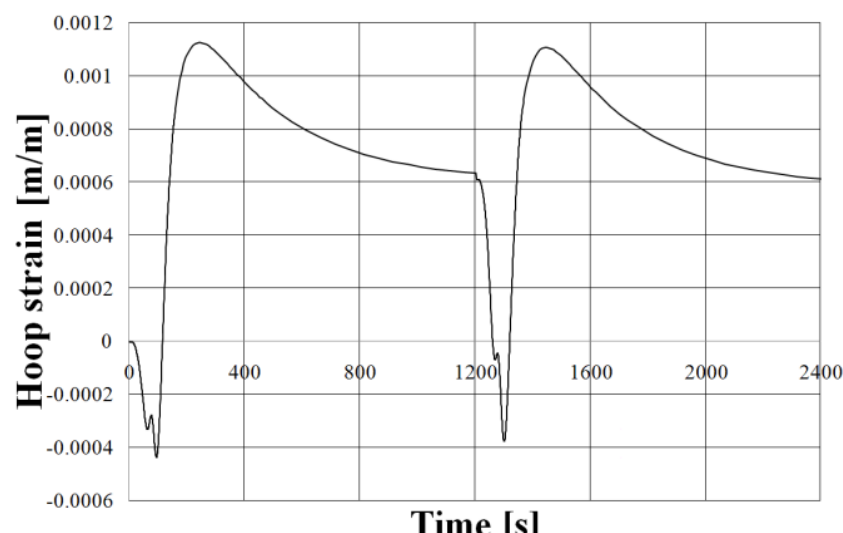

(a)

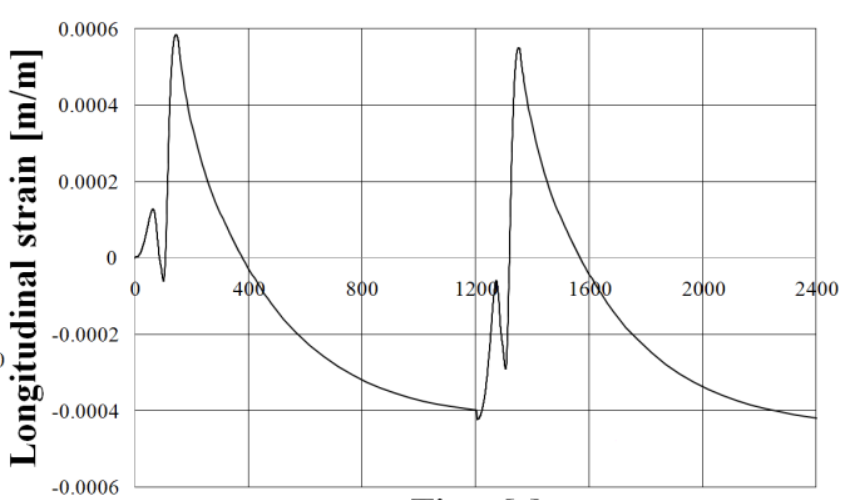

Time [s]

(b)

Fig. 4: Effect of welding residual stresses on the strain evolution during the second welding: (a) strain evolution in hoop direction; (b) strain evolution in the longitudinal direction.

The result of these simulations can be seen in Fig. 4. Again the strain evolution in hoop direction and longitudinal direction are plotted. In these strain evolutions, the two cycles are clearly visible. The first part of the strain evolution is the same as in Fig. 3, the curves without initial stress field. In this case, the strain evolution in both longitudinal and circumferential is affected, this is because the stress/strain state at the end of the first cycle contains stresses in both directions, see Fig. 2 (a), (b) and (c) and plastic strains in the area of the weld bead, Fig. 2 (d).

In the way the strain evolution is plotted here, it seems at first sight that there is no influence of the stress/strain state of the first welding cycle. However, when the stress/strain state after the first weld bead is taken as the reference state, it is clear that the starting point of the strain evolution in 
the hoop direction should be shifted over $-600 \mu \mathrm{m} / \mathrm{m}$ and the longitudinal strain evolution should be shifted over $+400 \mu \mathrm{m} / \mathrm{m}$.

\section{Conclusion and future work}

It is shown that starting the welding simulations with an initial stress field in hoop direction affects the evolution of the hoop strain observed during the welding. The stress field used in this analysis represents the bending moment that tends to open the tube when the cross section is cut in longitudinal direction. Imposing the thermal cycle corresponding to the welding twice to the initial stress free tube affects clearly the strain evolution observed during the welding both in longitudinal and circumferential direction. In this case, the strain evolution is influenced by the stress/strain state (involving all in plane stress components and plastic strains).

From the results presented here, it is clear that residual stresses, present in the tube from former production steps (rolling and welding) affect the strain evolution observed during the welding of the tube. This means that the simulations and experiments should be in agreement on this point. Either the tubes in the experimental setup should be annealed or the residual stresses as present in the actual tubes should be implemented in the simulations.

Future work will consist of performing welding experiments on annealed tubes which are considered stress-free on the one hand, and on the other hand simulating the rolling process of the tubes to discover the stress/strain state of the tube after rolling. This will allow extending the elastic stress state as described here with plastic strain state and stresses in longitudinal direction.

\section{References}

[1] M. Sutton, W. Wolters, W. Peters, W. Ranson and S. McNeill, Image and Vision Computing, Vol.1 (1983), p.133-139.

[2] T. Chu, W. Ranson, M. Sutton and W. Peters. Experimental Mechanics Vol. 25-3 (1985), p.232-244.

[3] M. Sutton, J. Orteu and H. Schreier: Image correlation for shape, motion and deformation measurements. Springer (2009).

[4] M. De Strycker, L. Schueremans, W. Van Paepegem and D. Debruyne, Optics and Lasers in Engineering, Vol. 48 (2010), p.978-986.

[5] M. De Strycker, P. Lava, W. Van Paepegem, L. Schueremans and D.Debruyne, "Measuring welding deformations with the Digital Image Correlation technique." submitted to the Welding Journal.

[6] ABAQUS Inc. ABAQUS version 6.9 Analysis User's Manual, (2009).

[7] M. De Strycker, D. Debruyne, W. Van Paepegem and L. Schueremans, Welding of cold-rolled steel tubes: sensitivity of fea input parameters, proceedings of Complas X, Barcelona (2009).

[8] L. Gardner and K.T. Ng. Fire Safety Journal, Vol. 41 (2006), p.185-203.

[9] K.W. Mahin, W. Winters, T.M. Holden, R.R. Hosbons and S.R. MacEwen, Welding Journal, Vol. 70 (1991), p.245s-260s.

[10]L. Gardner and N.R. Baddoo. Journal of Constructional Steel Research, Vol. 62 (2006), p.532543.

[11]L.E. Lindgren, Journal of Thermal Stresses, Vol. 24 (2001), p.195-231.

[12] J.A. Goldak and M. Akhlaghi. Computational welding mechanics. Springer (2005). 